\title{
Construction of a 4D Statistical Atlas of the Cardiac Anatomy and Its Use in Classification
}

\author{
Dimitrios Perperidis ${ }^{1}$, Raad Mohiaddin $^{2}$, and Daniel Rueckert ${ }^{1}$ \\ ${ }^{1}$ Visual Information Processing Group, Department of Computing, \\ Imperial College, London, 180 Queen's Gate, London SW7 2BZ, United Kingdom \\ ${ }^{2}$ Royal Brompton and Harefield NHS Trust, Sydney Street, London, United Kingdom
}

\begin{abstract}
In this paper we present a novel method for building a 4D statistical atlas describing the cardiac anatomy and how the cardiac anatomy changes during the cardiac cycle. The method divides the distribution space of cardiac shapes into two subspaces. One distribution subspace accounts for changes in cardiac shape caused by inter-subject variability. The second distribution subspace accounts for changes in cardiac shape caused by deformation during the cardiac cycle (i.e. intra-subject variability). Principal component analysis (PCA) have been performed in order to calculate the most significant modes of variation of each distribution subspace. During the construction of the statistical atlas we eliminate the need for manual landmarking of the cardiac images by using a nonrigid surface registration algorithm to propagate a set of pseudo-landmarks from an automatically landmarked atlas to each frame of all the image sequences. In order to build the atlas we have used 26 cardiac image sequences from healthy volunteers. We show how the resulting statistical atlas can be used to differentiate between cardiac image sequences from patients with hypertrophic cardiomyopathy and normal subjects.
\end{abstract}

\section{Introduction}

The early diagnosis and treatment of cardiovascular diseases is crucial in order to reduce mortality and to improve patients' quality of life. Recent advances in the development of magnetic imaging (MR) have enabled the acquisition of high resolution 4D cardiac image sequences which describe the cardiac anatomy as well as function. The acquisition of $4 \mathrm{D}$ cardiac image sequences drastically increases the amount of data to be interpreted by clinicians. Therefore, applications assisting the automatic interpretation of MR images are of high importance for increasing the clinical use of MR imaging.

A large number of approaches have been developed for the volumetric modeling of the heart. A comprehensive review of these approaches can be found in Frangi et al. [1]. Biomechanical models of the heart have been developed by combing surface information and motion information [2] and by using a deformation model inspired by continuum mechanics [3]. In contrast to these biomechanical models a number of researchers have developed statistical models (e.g. Active Shape Models) of the cardiac anatomy [4] [5] and statistical models of the appearance of the heart (e.g. Active Appearance Models) [6] [7]. For example, Frangi et al. have presented an approach for the construction of three-dimensional statistical shape models of the cardiac anatomy 
[8]. This approach eliminates the need for landmarking by using non-rigid registration to propagate landmarks from an automated landmarked atlas to the rest of the images. The resulting model includes the left and right ventricle. The approach developed by Lötjönen et al. goes one step further: In this work, statistical shape models of the atria, ventricles and epi-cardium from short-axis and long-axis MR images are constructed and used for the segmentation of cardiac images [9]. In addition a variety of methods which model shape variability have been explored including PCA, ICA and LPD. However, in both cases the statistical shape models describe only the 3D cardiac anatomy at a single time point and ignore the shape variation during the cardiac cycle. Although cardiac modeling of the anatomy is relatively well investigated, very few attempts have been made to build a computerized atlas which captures functional variability of the heart across a group of subjects. Rao et al. suggested a framework for building an atlas of the myocardial motion [10] by using tagged MR image sequences to calculate the cardiac motion. Then, the calculated motion fields of different subjects are mapped into the same coordinate system using a vector field transformation technique which accounts for differences in the size, orientation and shape of the heart.

In this paper we present a novel method for building a 4D statistical atlas describing the cardiac anatomy and how the cardiac anatomy changes during the cardiac cycle. In previous work we developed 4D probabilistic atlases of the left ventricle, the myocardium and the right ventricle describing the anatomy and function of a healthy heart [11]. While these probabilistic atlases contain information about the degree of variability at every voxel, statistical atlases provide additional information about the type of variability. The key contribution of our work is the construction of 4D statistical model of the heart that subdivides the distribution space of cardiac shapes into two subspaces: One distribution space accounts for changes in cardiac shape due to deformations throughout the cardiac cycle and the other distribution space accounts for the changes in cardiac shape due to variability across the population. Furthermore, we have used these statistical models to differentiate between cardiac image sequences from patients with hypertrophic cardiomyopathy and normal volunteers.

\section{Building a 4D Statistical Atlas}

Segmentation of Cardiac MR Image Sequences. The method developed by LorenzoValdés et al. [12] has been used to segment the image sequences. In this method the first frame of each image sequence is segmented manually and then the segmentation is propagated to the subsequent frames using a non-rigid registration algorithm. The images sequences are segmented into three anatomical structures: the left ventricle, the myocardium and the right ventricle. After segmenting the image sequences, shape based interpolation is used to resample the images to isotropic voxels of size $1 \mathrm{~mm} \times 1 \mathrm{~mm} \times 1 \mathrm{~mm}$.

Mapping the Image Sequences to the Same Spatio-Temporal Coordinate System. The second stage in the construction procedure of the atlas is to use a spatio-temporal registration method to align the image sequences into the same spatio-temporal coordinate system. The registration method is similar to one which we have previously 
introduced [13] and has also been used for the construction of a probabilistic atlas of the cardiac anatomy and function [11]. It uses a 4D mapping which has been resolved into decoupled spatial and temporal components $\mathbf{T}_{\text {spatial }}$ and $\mathbf{T}_{\text {temporal }}$ respectively.

The spatial transformation used is an affine transformation with 9 degrees of freedom which accounts for spatial differences caused by orientation, translation and scaling. The temporal transformation consists of a global part which scales the image sequences to match the end-systolic and end-diastolic time points and a local part which deforms the temporal characteristics of each image sequence to follow the same motion pattern with the reference image sequence. The local temporal transformation is modeled by a free-form deformation using a 1D B-spline:

$$
\mathbf{T}_{\text {temporal }}^{\text {local }}(t)=\sum_{l=0}^{3} B_{l}(u) \phi_{t_{i+l}}
$$

where $\Phi$ denotes a set of $n_{t}$ control points $\phi_{t}$ with a temporal spacing $\delta_{t}$ and $B_{l}$ represents the 1-th basis function of the B-spline. The optimal spatial and temporal transformation is found by maximising a voxel based similarity measure, the normalized mutual information (NMI). The NMI of two image sequences can be calculated directly from the joint intensity histogram of the two sequences over their spatio-temporal domain of overlap.

Building the Statistical Atlas of the Heart. After obtaining the spatio-temporal mappings, the segmented image sequences are transformed to the common spatio-temporal coordinate system. Then, the transformed images are blurred with a Gaussian kernel to compensate for low out-of-plane resolution of the images which results in significant partial volume effects in the segmentation. In our approach we eliminate the need for manual landmarking by using a method similar to the one introduced by Frangi et al.[8]. In this method, a set of pseudo-landmarks are propagated from an automatically landmarked atlas to all the frames of each image sequence.

Landmark Extraction and Propagation. After blurring the image sequences with a Gaussian kernel, the marching cubes [14] algorithm is used to generate a dense triangulation (pseudo-landmarks) of the boundary surfaces of each anatomical structure (the left ventricle, the myocardium and the right ventricle) of all the frames of each image sequence. In order to perform any statistical analysis correspondence between the pseudo-landmarks of each image needs to be established. This is achieved by using a surfaced based registration method based on B-Splines. The end-diastolic frame of the image sequence used as reference during the construction of the atlases is also used as the reference surface in these registrations. After registering all surfaces, we use the obtained transformations to propagate the pseudo-landmarks of the reference surface to each frame.

Modeling Shape Variability. Let $\left\{\mathbf{q}_{\mathbf{i k}} ; i=0 \ldots n_{p} ; k=0 \ldots n_{f}\right\}$ denote $n$ shapes ( $n_{p}$ subjects with $n_{f}$ frames each). Each shape consists of $m 3 \mathrm{D}$ landmarks, $\mathbf{p}_{\mathbf{j}}=$ $\left(p_{1 j}, p_{2 j}, p_{3 j} ; j=1 \ldots . m\right)$. Each vector $\mathbf{q}_{\mathbf{i k}}$ will consist of the landmarks $\left(p_{11}, p_{21}, p_{31}\right.$, $\left.p_{12}, p_{22}, p_{32}, \ldots, p_{1 m}, p_{2 m}, p_{3 m}\right)$. The aim of the statistical analysis is to approximate the distribution of the landmarks with a linear model of the form: 


$$
\mathbf{q}=\overline{\mathbf{q}}+\Phi \mathbf{b}
$$

where $\overline{\mathbf{q}}$ is the average landmark vector, $\mathbf{b}$ is the shape parameter vector of the model, and $\Phi$ is a matrix of eigen-vectors. The matrix $\Phi$ is obtained by performing a principal component analysis (PCA) to the covariance matrix $\mathbf{C}$. During the principal component analysis, the principal components of $\mathbf{C}$ are calculated as its eigenvectors $\phi_{i}$ and the corresponding eigenvalues $\lambda_{i}$ are also calculated (such that $\lambda_{i}<\lambda_{i+1}$ ).

The aim of our statistical analysis is to identify what changes in the cardiac anatomy occur due to the cardiac cycle and what changes occur due to shape variation across the population. Therefore, we want to use principal components analysis (PCA) to find the estimate of two subspaces of the overall distribution. In order to achieve this we perform two separate principal component analysis. The covariance matrices for the total shape distribution is given by:

$$
\mathbf{C}_{\text {total }}=\frac{1}{n_{f} n_{p}} \sum_{i=1}^{n_{p}} \sum_{k=1}^{n_{f}}\left(\mathbf{q}_{\mathbf{i k}}-\overline{\mathbf{q}}\right)\left(\mathbf{q}_{\mathbf{i k}}-\overline{\mathbf{q}}\right)^{T}
$$

where $n_{f}$ is the number of frames of each image sequence, $n_{p}$ the number of images sequences and $\overline{\mathbf{q}}$ is the mean shape.

The covariance matrix of the shape differences occurring due to the cardiac cycle is given by:

$$
\mathbf{C}_{\text {within }}=\frac{1}{n_{f} n_{p}} \sum_{i=1}^{n_{p}} \sum_{k=1}^{n_{f}}\left(\mathbf{q}_{\mathbf{i k}}-\overline{\mathbf{q}}_{\mathbf{i}}\right)\left(\mathbf{q}_{\mathbf{i k}}-\overline{\mathbf{q}}_{\mathbf{i}}\right)^{T}
$$

where $\overline{\mathbf{q}}_{\mathbf{i}}$ is the mean for the subject $i$ (the image sequences contain the same number of frames since they are registered in the temporal domain) and $\mathbf{q}_{\mathbf{i k}}$ is the shape of frame $k$ of subject $i$.

The covariance matrix which described the shape differences occurring across the population is given by:

$$
\mathbf{C}_{\text {between }}=\frac{1}{n_{p}} \sum_{i=1}^{n_{p}}\left(\overline{\mathbf{q}}_{\mathbf{i}}-\overline{\mathbf{q}}\right)\left(\overline{\mathbf{q}}_{\mathbf{i}}-\overline{\mathbf{q}}\right)^{T}
$$

where, as in eq. 4, $n_{p}$ is the number of image sequences and $\overline{\mathbf{q}}$ is the total mean.

In order to find the principal components of each subspace the eigen values and vectors of each covariance matrix (eq. 4 and 5) are calculated. A similar decomposition of the total distribution to subspaces has been used by Costen et al. for the automatic extraction of the face identity-subspace [15]. New shape examples can be generated by varying the parameters $b$ of equation 2. Assuming that the distribution of the data follows a multidimensional Gaussian distribution (this assumption has some limitations), the variance of the $i$ th parameter of $\mathbf{b}$ across the training set is given by $\lambda_{i}$. If we apply limits in the variation of $b_{i}$ such that $b_{i} \leq \pm 3 \sqrt{\lambda_{i}}$ we ensure the generated shape is similar to those contained in the training class.

\section{Results}

Materials. In order to produce the atlas we have acquired 26 untagged MR image sequences from healthy volunteers. The images have been acquired using a Siemens 
Sonata 1.5T scanner using TrueFisp pulse sequence in a form of a series short-axis images. Seven of the images were acquired using retrospective gating acquisition while the rest were acquired using prospective gating acquisition. One of the image sequences was selected to be the reference subject of the spatio-temporal registration. Care was taken to ensure that the reference subject was a normal representative of the population. The reference subject had in-plane resolution of $192 \times 256$ with pixel size of $1.48 \times$ $1.48 \mathrm{~mm}$ and a slice thickness of $10 \mathrm{~mm}$ (which is the typical pixel size used in these acquisitions). After the shape based interpolation the slice thickness was reduced to $1 \mathrm{~mm}$. The images covered the entire left-ventricle form base to apex.

Statistical Model of the Variability Across Subjects. These models describe the significant changes in the shape of the left ventricle, the myocardium due to subjects' different heart shape. Figure 1 shows the three most significant modes of variation for the left ventricle (a) and the myocardium (b). For the left ventricle, the three most significant modes of shape variation describe the differences in the size of the left ventricle (mode 1), the variation of the position of the apex of the heart (mode 2) and the elongation of the apex of the heart (mode 3 ). For the myocardium, the three most significant modes of variation describe the size of the myocardium (mode 1), the thickness of the myocardium (mode 2 ) and the direction of the myocardium long-axis (mode 3 ). In order to describe $90 \%$ of the shape variability of the inter-subject distribution subspace 13 (of 26) modes of shape variation are required.

Statistical Model of the Variability Across the Cardiac Cycle. These models describe the most significant changes in the shape of the left ventricle and the myocardium which occur due to the cardiac cycle. Figure 2 shows the three most significant modes of variation of the left ventricle (a) and the myocardium (b). For the left ventricle, the

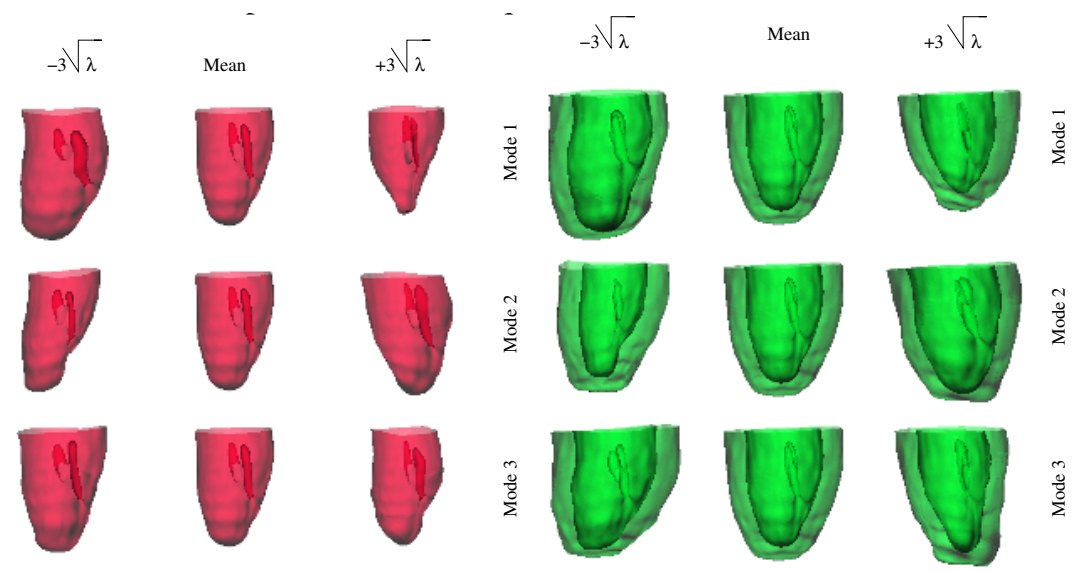

(a)

(b)

Fig. 1. The significant modes of variation across subjects of (a) left ventricle and (b) myocardium. Animations of all the atlases and also of atlases of the right ventricle can be found at: http:/www.doc.ic.ac.uk/ dp1/Research/StatisticalAtlases/ 


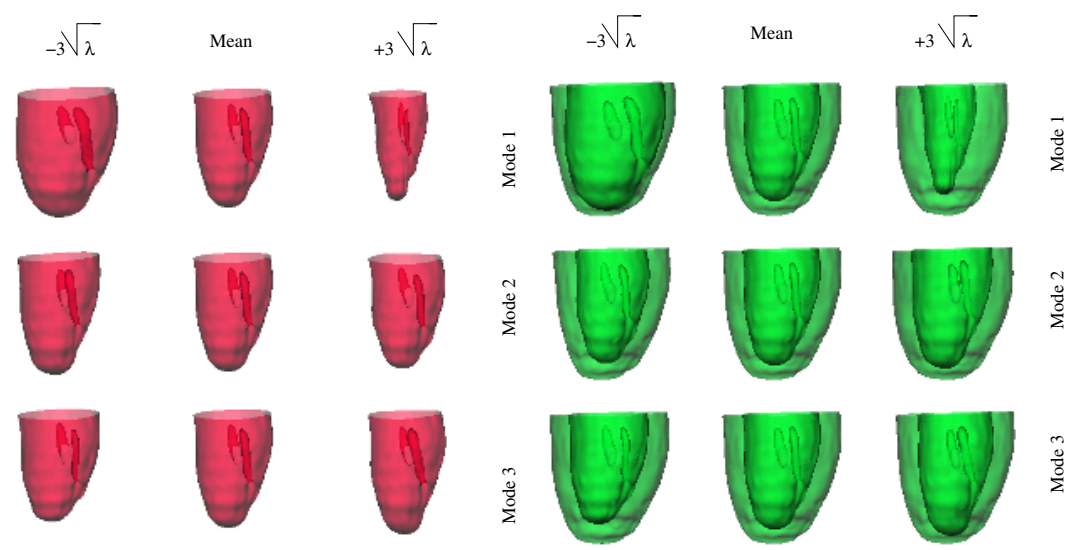

(a)

(b)

Fig. 2. The significant modes of variation across the cardiac cycle of (a) left ventricle and (b) myocardium

three most significant modes of variation (fig. 2(a)) describe the differences in the volume of the left ventricle during the cardiac cycle (mode 1), the twisting of the heart during the contraction phase (mode 2) and the changes in the position of the apex of the left ventricle and also the position of the papillary muscles (mode 3). For the myocardium, the three most significant modes of variation (fig. 2(b)) describe the changes in the size of the left ventricle and the thickening of the myocardium, the twisting of the myocardium during the contraction phase (mode 3 ) and the movement of the cardiac wall (mode 3). In order to describe $90 \%$ of the shape variability of the intra-subject distribution subspace 16 (of 468) modes shape variation are required.

\section{Classification Using the 4D Statistical Atlas}

In this section we demonstrate a possible use of the statistical atlases for the classification of cardiac data. We used the above statistical models to classify cardiac data from normal volunteers and patients with hypertrophic cardiomyopathy (a condition in which the myocardium has an excessive thickening). In order to perform this classification we have excluded six subjects from the model (i.e. the model has been constructed from only 20 healthy subjects) and we have acquired MR image sequences from 8 patients with hypertrophic cardiomyopathy. The same processing steps for the registration and pseudo-landmark extraction and propagation were used for these image sequences as for ones used for the construction of the statistical models (see section 2). Then, for each image sequence the mean surface (over the cardiac cycle) was calculated. These mean surfaces were projected into the shape space of the statistical models. Figure 3 shows the projections of the subjects' myocardium to the space of the across population atlas (a) and across cardiac cycle atlas (b). We clearly see from the distribution of the data that a simple classifier will enable the correct differentiation between normal and hypertrophic subjects. 


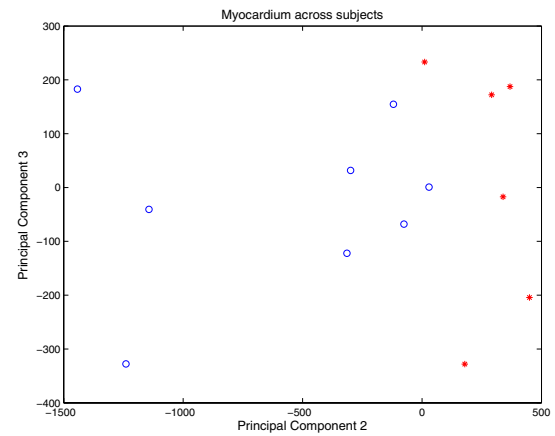

(a)

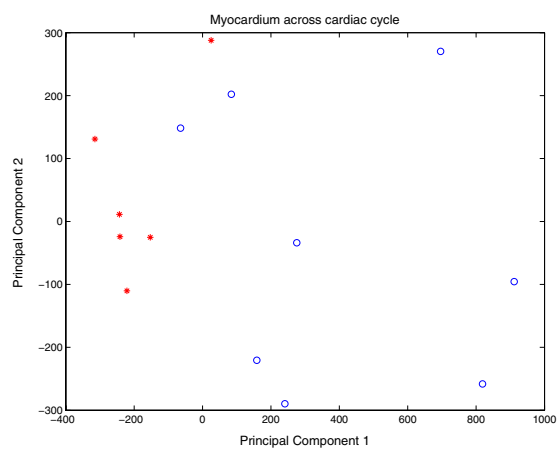

(b)

Fig. 3. Projection of the myocardium to the space of the (a) across the subjects atlas and (b) across the cardiac cycle atlas (the circles on the figures are the subjects with hypertrophic cardiomyopathy while the stars the normal ones)

Table 1. Accuracy of the classification using the statistical model of the myocardium describing changes in the anatomy due to the cardiac cycle (model A), due to inter-subject variation (model B) and a combination of both

\begin{tabular}{||l||c|c|c||}
\hline & Model A & Model B & Model A and B \\
\hline Normal & $83 \%$ & $100 \%$ & $100 \%$ \\
\hline Hypertrophic cardiomyopathy & $100 \%$ & $87.5 \%$ & $87.5 \%$ \\
\hline
\end{tabular}

In order to classify the data we used a $k$-weighted $N N$-classifier. A leave one out experiment was performed. The first 4 principal components were employed when using the statistical atlas describing the cardiac shape variability due to cardiac cycle, while the second and the third principal components were employed when using the statistical atlas describing the inter-subject shape variability. In this case, the first principal component was not used in the classification since it describes the size of the myocardium in the base to apex direction. Furthermore, the combination of these principal components (from both models) were also used for data classification. The classification results are reported in table 1

\section{Conclusions}

In this paper we presented a novel method for building a 4D statistical atlas describing the cardiac anatomy and how the cardiac anatomy changes during the cardiac cycle. Contrary to probabilistic atlases, the statistical atlases provide not only information regarding how much variability exists in the data but also what the variability is. In order to build the atlas we have used 26 cardiac image sequences from normal volunteers. The method separates the distribution space of the cardiac shape into two subspaces. One distribution subspace accounts for the changes in cardiac shape caused by inter-subject variability. The second distribution subspace accounts for the changes in cardiac shape 
caused by deformation in the cardiac cycle (i.e. intra-subject variability). Principal component analysis (PCA) has been performed in order to calculate the most significant modes of variation of each distribution subspace. Moreover, our method eliminates the need for manual landmarking of the cardiac images by using a non-rigid registration algorithm to propagate landmarks from an from an automatically landmarked to each image. We have also demonstrated a possible use of the statistical atlases by using them to differentiate between cardiac image sequences from patients with hypertrophic cardiomyopathy and normal subjects.

\section{References}

1. A. F. Frangi, W. J. Niessen, and M. A. Viergever. Three-dimensional modeling for functional analysis of cardiac images: A review. IEEE Transactions on Medical Imaging, 20(1):2-25, 2001.

2. P. C. Shi, A. J. Sinusas, T. Constable, and J. S. Duncan. Volumetric deformation analysis using mechanics-based data fusion: Applications in cardiac motion recovery. International Journal of Computer Vision, 35(1):87-107, 1999.

3. X. Papademetris. Estimation of 3D Left Ventricular Deformation from Medical Images Using Biomechanical Models. PhD thesis, Yale University, 2000.

4. J. Lötjönen, J. Koikkalainen, D. Smutek, S. Kivistö, and K. Lauerma. Four-chamber 3D statistical shape model from cardiac short-axis MR images. In Sixth Int. Conf. on Medical Image Computing and Computer-Assisted Intervention (MICCAI '03), number 2878 in Lecture Notes in Computer Science, pages 491-498. Springer, 2003.

5. P. Horkaew and G. Z. Yang. Optimal deformable surface models for 3D medical image analysis. In Information Processing in Medical Imaging: Proc. 18th International Conference (IPMI’03), number 2732 in Lecture Notes in Computer Science, pages 13-24. Springer, 2003.

6. S. C. Mitchell, B. P. F. Lilieveldt, R. J. van der Geest, H. G. Bosch, J. H. C. Reiber, and M. Sonka. Multistage hybrid active appearance model matching: Segmentation of left and right ventricles in cardiac MR images. IEEE Transactions on Medical Imaging, 20(5):415523, 2001.

7. S. C. Mitchell, J. G. Bosch, B. P. F. Lelieveldt, R.J . van der Geest, J. H. C. Reiber, and M. Sonka. 3D active appearance models:segmentation of cardiac MR and ultrasound images. IEEE Transactions on Medical Imaging, 21(9):1167-1178, 2002.

8. A. F. Frangi, D. Rueckert, J. A. Schnabel, and W. J. Niessen. Automatic construction of multiple-object three-dimensional statistical shape models: Application to cardiac modeling. IEEE Transaction on Medical Imaging, 21(9):1151-1165, 2002.

9. J. Lötjönen, S. Kivistö, J. Koikkalainen, D. Smutek, and K. Lauerma. Statistical shape model of atria, ventricles and epicardium from short- and long-axis MR images. Medical Image Analysis, 8(3):371-386, 2004.

10. A. Rao, G. I. Sanchez-Ortiz, R. Chandrashekara, M. Lorenzo-Valdés, R. Mohiaddin, and D. Rueckert. Comparison of cardiac motion across subjects using non-rigid registration. In Fifth Int. Conf. on Medical Image Computing and Computer-Assisted Intervention (MICCAI '02), number 2488 in Lecture Notes in Computer Science, pages 722-729. Springer, 2002.

11. D. Perperidis, M. Lorenzo-Valdés, R. Chandrashekara, A. Rao, R. Mohiaddin, G. I. SanchezOrtiz, and D. Rueckert. Building a 4D atlas of the cardiac anatomy and motion using MR imaging. In 2004 IEEE International Symposium on Biomedical Imaging: From Nano to Macro, 2004. 
12. M. Lorenzo-Valdés, G. I. Sanchez-Ortiz, A. Elkington, R. Mohiaddin, and D. Rueckert. Segmentation of 4D cardiac MR images using a probabilistic atlas and the EM algorithm. Medical Image Analysis, 8(3):255-265, 2004.

13. D. Perperidis, R. Mohiaddin, and D. Rueckert. Spatio-temporal free-form registration of cardiac MR image sequences. In Seventh Int. Conf. on Medical Image Computing and Computer-Assisted Intervention (MICCAI '04), number 3216 in Lecture Notes in Computer Science, pages 911-919. Springer, 2004.

14. W. E. Lorensen and H. E. Cline. Marching cubes: a high resolution 3D surface reconstruction algorithm. In Computer Graphics: SIGGRAPH'87 Conference Proceedings, volume 21, pages 163-169, 1987.

15. N. P. Costen, T .F. Cootes, G. J. Edwards, and C. J. Taylor. Automatic extraction of the face identity-subspace. Image and Vision Computing, 20(5-6):319-329, 2002. 